\title{
Pediatric-specific biomarkers: an important but challenging field
}

"...this issue of Biomarkers in Medicine highlights the importance and difficulties of the discovery, development and validation of biomarkers in pediatric patients and the future of the field."

In this issue of Biomarkers in Medicine, the themed content focuses on biomarkers in the pediatric setting. 'Differences in factors, such as the etiology, pathogenesis and treatment of pediatric disease, warrant the development of biomarkers for use in pediatrics, as biomarkers developed in adults may not be relevant.' Agerelated and size characteristics may also prevent the use of such biomarkers in pediatric patients. However, the development of biomarkers in pediatrics faces challenges; for example, it may be difficult to develop such biomarkers due to ethical considerations and low disease prevalence, which leads to low sample sizes [1]. This issue of Biomarkers in Medicine includes reviews discussing the development of biomarkers in various aspects of the pediatric field, and presents a preliminary communication article examining a potential biomarker in neonates.

In a previous issue of Biomarkers in Medicine, Goldman et al. discussed the scarcity of data regarding validated pediatric biomarkers, highlighting how biomarkers validated in adults and extrapolated to pediatrics can have issues due to the different pathogenesis of diseases in pediatrics and the effect of ontogeny on disease evolution and therapeutic response [2].

With such challenges in mind, Buijs et al. bring together literature concerning biomarker development in the pediatric intensive care unit, specifically focusing on biomarkers in cardiovascular monitoring, acute kidney injury, drug withdrawal and delirium [3]. The authors discuss the potential of biochemical substances, physiological signs and rating scores as possible biomarkers in the pediatric field, commenting on the rationale for selected biomarkers, studies performed in the pediatric intensive care unit, biomarker validation and the potential of the discussed biomarkers in drug trials and the clinic. They also speculate how advancing technologies, such as microarrays and proteomics, could further tailor drug therapy in the intensive care unit, citing studies on sepsis and septic shock where use of microarrays and bioinformatics has highlighted potential biomarkers whose expression profiles differ with age.

Sigdel et al. have further discussed the role of proteomics in pediatric medicine, focusing on the role of peptides and proteins as biomarkers for pediatric transplantation of the kidney, liver, heart and lungs, specifically in relation to mass spectrometry-based proteomics [4]. This review discusses the current status of research in this field, and the problems faced regarding the discovery of biomarkers for monitoring transplanted organs. The authors also highlight the need for standardization of sample collection, processing and storage in order to eliminate confounding factors; for the establishment of biorepositories for collection of large quantities of samples at regular intervals; and for improved collaborations in 'omics' research in biomarker discovery and development.

\section{"Differences in factors, such as the etiology, pathogenesis and treatment of pediatric disease, warrant the development of biomarkers for use in pediatrics, as biomarkers developed in adults may not be relevant."}

In the final review, Kwiatkowski et al. discuss the discovery of biomarkers for acute kidney injury in pediatric cardiac patients, highlighting how this group of patients may be more suitable for study during biomarker development compared with adult patients, as they have a planned period of renal ischemia and lack comorbidities, such as advanced age, diabetes and other diseases often seen in adult patients [5]. The authors continue on to discuss the properties of an ideal biomarker for acute kidney injury, promising candidate biomarkers of acute kidney injury such as NGAL and IL-18, studies directly comparing biomarkers,

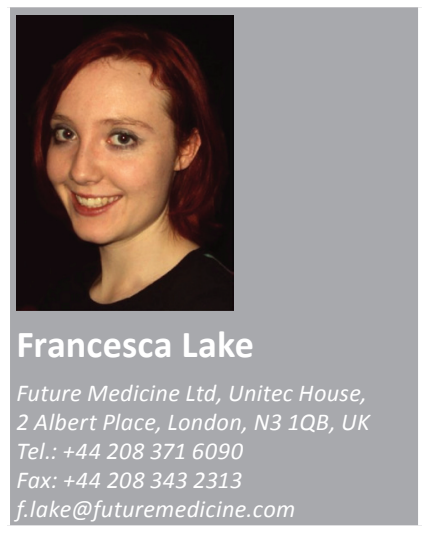

Future $\because \%$
Medicine $\%$ part of 
and biomarker panels, which, it is hoped, will enhance both the sensitivity and specificity of diagnosis and prognosis of acute kidney injury.

Also included in this themed issue is a preliminary communication by Smits et al. In this original research article, the authors aimed to study whether indirect bilirubin could act as a biomarker of reduced propofol clearance in neonates. Propofol is an anesthetic that is metabolized in the liver. It has been documented that the metabolization pathway of propofol is different in adults and neonates. The authors therefore feel that determination of new covariates of propofol clearance will help clinicians use this anesthetic safely in neonatal patients [6].

In summary, this issue of Biomarkers in Medicine highlights the importance and difficulties of the discovery, development and validation of biomarkers in pediatric patients and the future of the field.

\section{References}

1 Savage WJ, Everett AD. Biomarkers in pediatrics: children as biomarker orphans. Proteomics Clin. Appl. 4(12), 915-921 (2010).

2 Goldman J, Becker ML, Jones B, Clements M, Leeder JS. Development of biomarkers to optimize pediatric patient management: what makes children different? Biomarkers Med. 5(6), 781-794 (2011).
3 Buijs EA, Zwiers AJ, Ista E, Tibboel D, de Wildt SN. Biomarkers and clinical tools in critically ill children; toward tailored drug therapy? Biomarkers Med. 6(3), 239-257 (2012).

4 Sigdel TK, Gao X, Sarwal MM. Protein and peptide biomarkers in pediatric organ transplantation. Biomarkers Med. 6(3), 259-271 (2012).
$5 \quad$ Kwiatkowski DM, Goldstein SL, Krawczeski C. Biomarkers of acute kidney injury in pediatric cardiac patients. Biomarkers Med. 6(3), 273-282 (2012).

6 Smits A, De Cock RF, Cossey V, Knibbe CA, Allegaert K. Is indirect hyperbilirubinaemia a useful biomarker of reduced propofol clearance in neonates? Biomarkers Med. 6(3), 283-289 (2012). 